\title{
Strongyloides stercoralis and other intestinal parasites in patients receiving immunosuppressive drugs in northern Iran: a closer look at risk factors
}

\author{
Leila Mirzaei', Keyhan Ashrafi', Zahra Atrkar Roushan², Mohammad Reza Mahmoudi', \\ Irandokht Shenavar Masooleh', Behnaz Rahmati', Farshid Saadat ${ }^{1}$, Hamed Mirjalali', \\ Meysam Sharifdini ${ }^{1}$ \\ 'Department of Microbiology, School of Medicine, Guilan University of Medical Sciences, Rasht, Iran; ${ }^{2}$ Department of Biostatistics, Faculty \\ of Medicine, Guilan University of Medical Sciences, Rasht, Iran; ${ }^{3}$ Rheumatology Research Center, Razi Hospital, School of Medicine, Guilan \\ University of Medical Sciences, Rasht, Iran; ${ }^{4}$ Foodborne and Waterborne Diseases Research Center, Research Institute for Gastroenterology and \\ Liver Diseases, Shahid Beheshti University of Medical Sciences, Tehran, Iran
}

OBJECTIVES: The objective of this study was to evaluate the prevalence of Strongyloides stercoralis and other intestinal parasites in patients receiving immunosuppressive drugs in northern Iran and to investigate related risk factors.

METHODS: This cross-sectional study was conducted among 494 patients receiving immunosuppressive drugs, including cancer patients undergoing chemotherapy $(n=188)$ and those treated with prolonged corticosteroid administration $(n=306)$. All fresh fecal samples were examined using the direct wet-mount, formalin ethyl acetate concentration, and agar plate culture techniques.

RESULTS: In total, $16.8 \%$ of patients were positive for at least 1 intestinal parasite; the helminthic and protozoan infection rates were $5.1 \%$ and $12.3 \%$, respectively. The infection rate was significantly higher in corticosteroid-treated individuals (19.6\%) than cancer patients $(12.2 \%)(\mathrm{p}<0.05)$. The prevalence rate of $S$. stercoralis among patients receiving chemotherapy and those treated with corticosteroids were $4.3 \%$ and $5.2 \%$, respectively. The prevalence rate of S. stercoralis infection was significantly higher in older patients $(\mathrm{p}<0.05)$.

CONCLUSIONS: Strongyloidiasis is one of the most common parasites among patients receiving immunosuppressive drugs in northern Iran. Early diagnosis and proper treatment of these patients are necessary to minimize the complications of severe strongyloidiasis.

KEY WORDS: Strongyloidiasis, Immunocompromised patients, Risk factors, Iran

\section{Correspondence: Meysam Sharifdini}

Department of Microbiology, School of Medicine, Guilan University of Medical Sciences, Tehran Road Km6, Rasht 3363, Iran

E-mail: sharifdini@gums.ac.ir

Received: Dec 13, 2020 / Accepted: Jan 20, 2021 / Published: Jan 20, 2021

This article is available from: https://e-epih.org/

(c) This is an open-access article distributed under the terms of the Creative Commons Attribution License (https://creativecommons.org/licenses/by/4.0/), which permits unrestricted use, distribution, and reproduction in any medium, provided the original work is properly cited.

(C) 2021, Korean Society of Epidemiology

\section{INTRODUCTION}

Intestinal parasitic infections (IPIs) are major causes of significant morbidity and mortality rates worldwide. Many of these infections occur in developing and poor countries due to insufficient access to health services, malnutrition, and poor sanitation [1]. Moreover, the number of people with immune deficiencies continues to increase yearly due to the spread of human immunodeficiency virus (HIV) and the prescriptions of immunosuppressive medications such as corticosteroids and chemotherapy drugs for autoimmune diseases [2]. These groups of patients are 
at a particularly high-risk for severely complicated infections with some parasitic agents.

Strongyloides stercoralis is a soil-transmitted helminth estimated to infect about 370 million people worldwide, especially in tropical and subtropical countries [3]. It is transmitted through the penetration of infective larvae into human skin when in contact with soil. Strongyloidiasis has variable manifestations, ranging from asymptomatic cases to serious clinical syndromes such as hyperinfection and disseminated syndrome. Most infected individuals are completely asymptomatic, while some have mild gastrointestinal, pulmonary, and cutaneous symptoms with or without fever $[4,5]$. Chronic strongyloidiasis may change to severe, complicated, and deadly strongyloidiasis in patients receiving corticosteroid and other immunosuppressive treatment, as well as those with diseases such as diabetes, hematologic malignancies, HIV infection, and human T-lymphotropic virus type 1 infection. Several parasitological methods have been used to detect S. stercoralis larvae in stool samples, and multiple studies have confirmed that nutrient agar plate culture is more sensitive than other parasitological techniques [6,7]. The incidence of severely complicated strongyloidiasis has dramatically increased over the 2 recent decades, mostly due to the growing number of immunocompromised patients [8]. Therefore, rapidly diagnosing chronic infections, updating epidemiological information, and screening people at risk are helpful measures to reduce the mortality and morbidity rate of strongyloidiasis [6,9].

S. stercoralis is endemic in the northern and southern coastal provinces of Iran due to the suitable moist environment, climatic, and geographic factors for the establishment of its life cycle $[6,7,9$, 10]. Few studies have investigated infections of intestinal parasites, especially S. stercoralis, among immunosuppressed patients in Iran [11-13]. This study aimed to determine the frequency of $S$. stercoralis and other intestinal parasites among patients receiving immunosuppressive drugs in Guilan Province, in northern Iran, to provide a clear image of the current status of infections among these patients in this region.

\section{MATERIALS AND METHODS}

\section{Study area and sample collection}

Guilan Province is located along the southern part of the Caspian Sea in the north of $\operatorname{Iran}\left(36^{\circ} 34^{\prime}-38^{\circ} 27^{\prime} \mathrm{N}, 48^{\circ} 53^{\prime}-50^{\circ} 34^{\prime} \mathrm{E}\right)$ (Figure 1). This region has a humid subtropical climate. The average annual precipitation, relative humidity, and annual temperature are about $1,506 \mathrm{~mm}, 80 \%$, and $15.8^{\circ} \mathrm{C}$, respectively. This province is geographically divided into coastal plains and mountainous forest regions $[14,15]$. The most common jobs of rural residents are rice and tea cultivation and animal husbandry, which can expose them to soil-transmitted and zoonotic helminths.

This cross-sectional study was conducted among 494 patients receiving immunosuppressive drugs, including cancer patients undergoing chemotherapy $(\mathrm{n}=188)$ and those treated with prolonged corticosteroid administration $(n=306)$ from February 2018 to January 2019. Fresh stool samples were collected from referral hospitals in Guilan Province, including Razi, Rasool Akram, and Aria. Demographic data such as patients' sex, age, job, and educational level were recorded through interviews.

\section{Stool examinations}

All fresh fecal samples were examined using the direct wet-mount and formalin ethyl acetate concentration (FEC) techniques to detect the presence of any parasite. In addition, the agar plate culture (APC) technique was used to detect $S$. stercoralis infection, as described previously $[7,9,10]$. Briefly, about $3 \mathrm{~g}$ of a fresh stool sam-

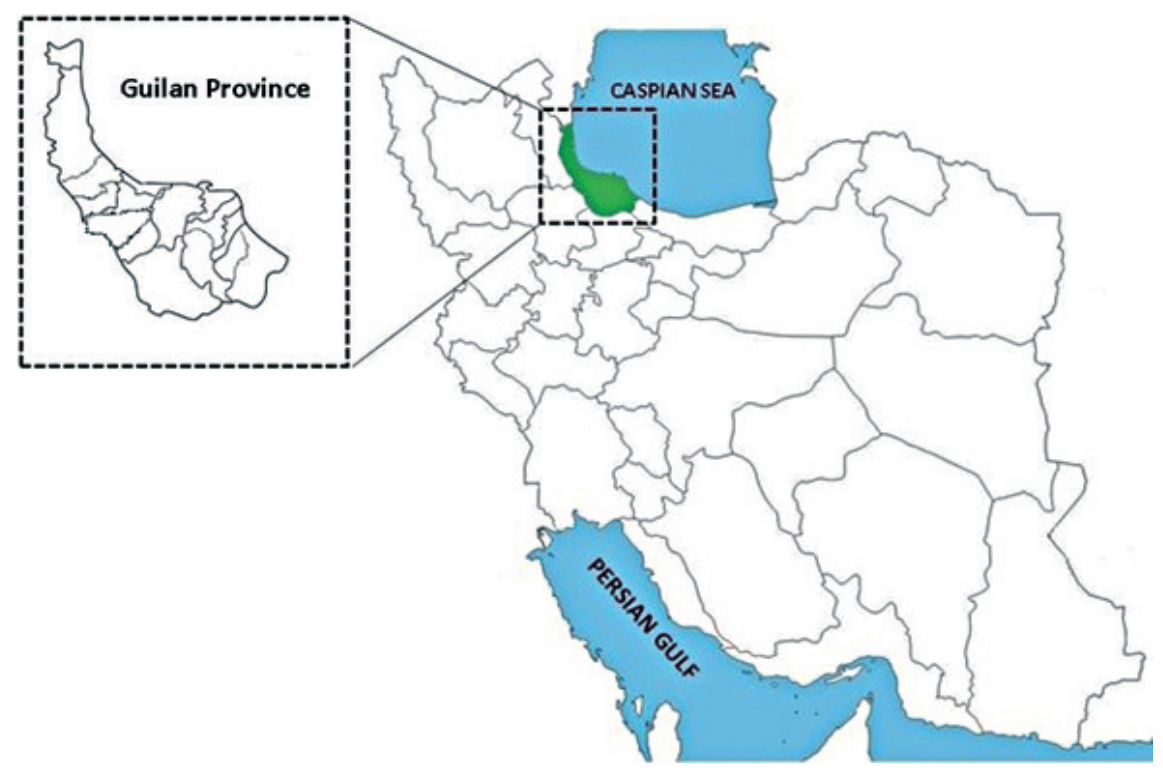

Figure 1. Map of Iran showing the geographical location of Guilan Province in northern Iran. 
ple was placed onto a nutrient agar plate and then incubated at room temperature for 2-3 days. Each plate was observed by stereomicroscopy to detect larvae and adult nematodes or their tracks. If the agar plate was positive, their surface was washed out by warm phosphate-buffered saline solution. The morphological characteristics of the parasites were then evaluated to identify and differentiate S. stercoralis from other possible nematodes such as Trichostrongylus spp., hookworms, and free-living nematodes $[7,9,10]$.

The parasitic loads of S. stercoralis-infected patients were categorized into 3 groups: (1) low infection: FEC-negative with 1-4 larvae counted on the agar plate surface, (2) moderate infection: FEC-positive with 5-10 larvae counted on the agar plate surface, (3) high infection: FEC-positive with more than 10 larvae counted on the agar plate surface $[7,9]$.

\section{Statistical analysis}

Data processing and analysis were performed using SPSS version 18.0 (SPSS Inc., Chicago, IL, USA), employing the chi-square and Fisher exact tests. A p-value of less than 0.05 was considered to indicate statistical significance.

\section{Ethics statement}

The protocol of this study was approved by the Ethics Committee of Guilan University of Medical Sciences, Iran (Ref. No. IR. GUMS.REC.1396.192).

Table 1. Prevalence of infections with intestinal parasites in patients receiving immunosuppressive drugs in Guilan Province, northern Iran, according to demographic factors

\begin{tabular}{|c|c|c|c|c|}
\hline Variables & $\begin{array}{c}\text { Positive, } \\
\text { n (\%) }\end{array}$ & $\begin{array}{c}\text { Negative, } \\
\text { n (\%) }\end{array}$ & OR $(95 \% \mathrm{Cl})$ & $\mathrm{p}$-value \\
\hline Age (yr) & & & & 0.669 \\
\hline$<40$ & $12(13.8)$ & $75(86.2)$ & 1.00 (reference) & \\
\hline $40-60$ & $43(18.1)$ & 195 (81.9) & $0.80(0.38,1.67)$ & \\
\hline$>60$ & $28(16.6)$ & $141(83.2)$ & $1.11(0.65,1.87)$ & \\
\hline Sex & & & & 0.399 \\
\hline Female & $41(15.4)$ & $225(84.6)$ & 1.00 (reference) & \\
\hline Male & $42(18.4)$ & $186(81.6)$ & $0.80(0.50,1.29)$ & \\
\hline Educational status & & & & 0.672 \\
\hline Illiterate & $23(17.6)$ & $108(82.4)$ & 1.00 (reference) & \\
\hline Under diploma & $43(17.7)$ & $200(82.3)$ & $1.29(0.65,2.55)$ & \\
\hline Diploma and above & $17(14.1)$ & $103(85.8)$ & $1.30(0.70,2.39)$ & \\
\hline Occupation & & & & 0.676 \\
\hline Farmer & $16(19.8)$ & $65(80.2)$ & 1.00 (reference) & \\
\hline $\begin{array}{c}\text { Government } \\
\text { employee }\end{array}$ & $2(9.5)$ & $19(90.5)$ & $1.02(0.50,2.11)$ & \\
\hline Worker & $9(13.6)$ & $57(86.4)$ & $0.44(0.09,2.03)$ & \\
\hline Housewife & $34(16.0)$ & $178(84.0)$ & $0.66(0.28,1.53)$ & \\
\hline Other & $22(19.3)$ & $92(80.7)$ & $0.79(0.44,1.44)$ & \\
\hline Location & & & & 0.227 \\
\hline City & $33(14.4)$ & $196(85.6)$ & 1.00 (reference) & \\
\hline Village & 50 (18.9) & $215(81.1)$ & $0.72(0.44,1.17)$ & \\
\hline
\end{tabular}

$\mathrm{OR}$, odds ratio; $\mathrm{Cl}$, confidence interval.

\section{RESULTS}

A total of 494 patients receiving immunosuppressive drugs were included, of whom 228 (46.2\%) were male and $266(53.8 \%)$ were female. In total, $83(16.8 \%)$ patients were positive for at least 1 intestinal parasite, and the helminthic and protozoan infection rates were $5.1 \%$ and $12.3 \%$, respectively. The prevalence rates of the parasites in cancer patients and corticosteroid-treated individuals were $12.2 \%$ and $19.6 \%$, respectively. Statistical analysis revealed a significant difference between these 2 groups in terms of the presence of IPIs $(\mathrm{p}=0.03)$. The distribution of IPIs according to sex, age group, educational status, location, and occupation is illustrated in Table 1. No significant difference between these demographic factors and the presence of IPIs was found.

The most prevalent parasites were Blastocystis hominis (10.3\%) and S. stercoralis (4.9\%). The prevalence rate of these parasites is shown in Table 2 . Mixed infections were observed in 6 individuals $(1.2 \%)$.

The prevalence rates of $B$. hominis in cancer patients and corticosteroid-treated individuals were $6.4 \%$ and $12.7 \%$, respectively, which constituted a significant difference $(\mathrm{p}=0.03)$.

S. stercoralis was detected in 24 patients by at least one parasitological method (Figure 2). The prevalence rates of $S$. stercoralis among patients receiving chemotherapy and those treated with corticosteroids were $4.3 \%$ and $5.2 \%$, respectively. No statistically significant difference was observed between the 2 groups regarding the prevalence of strongyloidiasis.

The prevalence of infection with $S$. stercoralis in the study population according to sex, age group, educational status, location, and occupation is illustrated in Table 3. A statistically significant difference in S. stercoralis infection according to age group $(\mathrm{p}<0.05)$. Other demographic characteristics failed to show any significant associations with the prevalence of $S$. stercoralis infection.

Among S. stercoralis-positive individuals, 7 (29.1\%), 14 (58.3\%), and $24(100.0 \%)$ cases were detected by direct wet mount, FEC, and APC, respectively. Therefore, the APC method showed a 3.4 times and 1.7 times higher ability to detect the parasite than the direct wet-mount and FEC techniques, respectively.

Table 2. Prevalence of infections with different species of intestinal parasites in patients receiving immunosuppressive drugs in Guilan Province, northern Iran

\begin{tabular}{llr}
\hline Intestinal parasites & $\mathrm{n}(\%)$ \\
\hline \multirow{2}{*}{ Single } & Giardia lamblia & $4(0.8)$ \\
& Entamoeba coli & $6(1.2)$ \\
& Blastocystis hominis & $46(9.3)$ \\
& Trichostrongylus spp. & $1(0.2)$ \\
& Strongyloides stercoralis & $20(4.0)$ \\
Double & Blastocystis hominis+Strongyloides stercoralis & $3(0.6)$ \\
& Blastocystis hominis+Giardia lamblia & $1(0.2)$ \\
& Blastocystis hominis+Entamoeba coli & $1(0.2)$ \\
& Trichostrongylus spp.+Strongyloides stercoralis & $1(0.2)$ \\
\hline
\end{tabular}



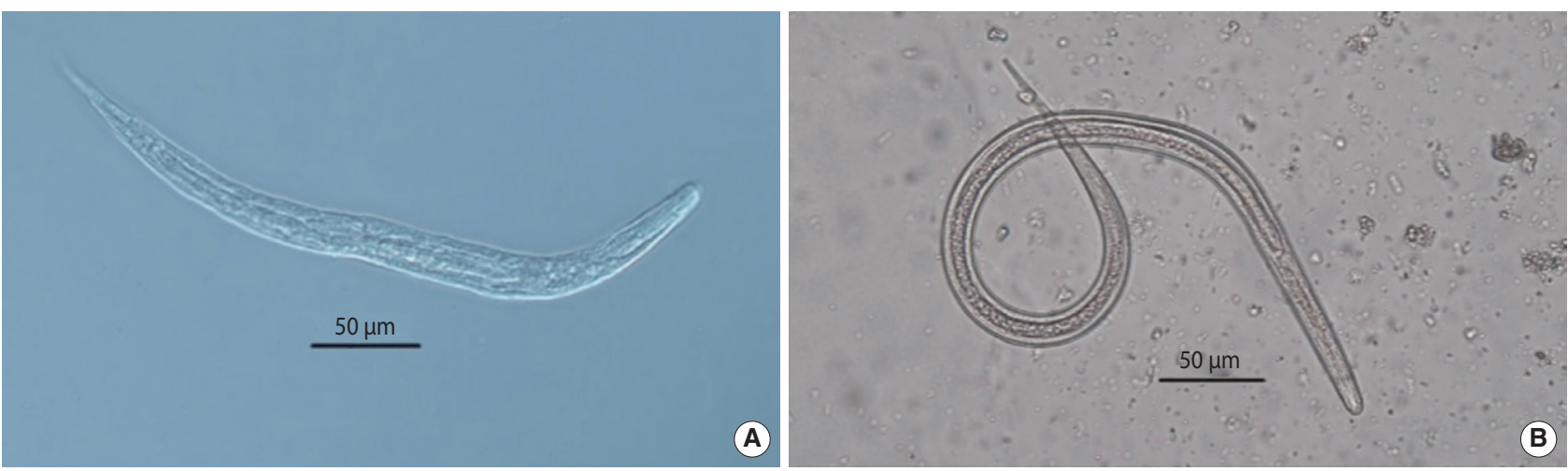

Figure 2. Rhabditiform (A) and filariform (B) larvae of Strongyloides stercoralis obtained through agar plate culture.

Table 3. Prevalence of infections with Strongyloides stercoralis in patients receiving immunosuppressive drugs in Guilan Province, northern Iran, according to demographic factors

\begin{tabular}{|c|c|c|c|c|}
\hline Variables & $\begin{array}{c}\text { Positive, } \\
\text { n (\%) }\end{array}$ & $\begin{array}{c}\text { Negative, } \\
\text { n (\%) }\end{array}$ & OR $(95 \% \mathrm{Cl})$ & $\mathrm{p}$-value \\
\hline Age (yr) & & & & 0.020 \\
\hline$<40$ & $1(1.1)$ & $86(98.9)$ & $0.12(0.17,0.99)$ & \\
\hline $40-60$ & $9(3.8)$ & $229(96.2)$ & $0.43(0.18,1.03)$ & \\
\hline$>60$ & $14(8.3)$ & $155(91.7)$ & 1.00 (reference) & \\
\hline Sex & & & & 0.530 \\
\hline Female & $11(4.1)$ & $255(95.9)$ & 1.00 (reference) & \\
\hline Male & $13(5.7)$ & $215(94.3)$ & $0.71(0.31,1.62)$ & \\
\hline Educational status & & & & 0.088 \\
\hline Illiterate & $11(8.4)$ & $120(91.6)$ & 1.00 (reference) & \\
\hline Under diploma & $9(3.7)$ & $234(96.3)$ & $2.65(0.82,8.58)$ & \\
\hline Diploma and above & $4(3.3)$ & $116(96.7)$ & $1.11(0.33,3.69)$ & \\
\hline Occupation & & & & 0.080 \\
\hline Farmer & $9(11.1)$ & $72(88.9)$ & 1.00 (reference) & \\
\hline $\begin{array}{c}\text { Government } \\
\text { employee }\end{array}$ & $1(4.8)$ & $20(95.2)$ & $2.72(0.87,8.46)$ & \\
\hline Worker & $1(1.5)$ & $65(98.5)$ & $1.09(0.12,9.83)$ & \\
\hline Housewife & $8(3.8)$ & $204(96.2)$ & $0.33(0.03,2.93)$ & \\
\hline Other & $5(4.4)$ & 109 (95.6) & $0.85(0.27,2.67)$ & \\
\hline Location & & & & 0.408 \\
\hline City & $9(3.9)$ & $220(96.1)$ & 1.00 (reference) & \\
\hline Village & $15(5.7)$ & $250(94.3)$ & $0.68(0.29,1.58)$ & \\
\hline
\end{tabular}

OR, odds ratio; $\mathrm{Cl}$, confidence interval.

In the positive cases, low, moderate, and high infection rates were detected in 10 (41.7\%), 7 (29.2\%), and 7 (29.2\%) cases, respectively. No significant relationship was demonstrated between the parasitic load and sex, age group, and the 2 groups of immunosuppressed patients.

\section{DISCUSSION}

Immunocompromised patients are at risk of serious and deadly infections with intestinal parasites in many countries, particularly in tropical and subtropical developing countries. In the current study, $16.8 \%$ of people taking immunosuppressive drugs in Guilan Province, northern Iran, were infected with intestinal parasites. Moreover, the overall prevalence of IPIs among corticosteroidtreated patients was significantly higher than that among cancer patients. It is now well established that both humoral and cellular immune functions are related to the psycho-neuroendocrine axis, in which any interaction via a prescribed corticosteroid disrupts immune responses against a parasite [16].

The prevalence rate of IPIs in cancer patients was $12.2 \%$, which is higher than that previously reported for central Iran (6.7\%) [17] and northwest Iran (10\%) [18], yet significantly lower than that reported for Tehran, Iran (25.9\%) [19], Yemen (63.1\%) [20], southern Brazil (61.6\%) [21], and Egypt (85.5\%) among cancer therapy recipients with concurrent diarrhea [22].

Few studies have investigated the frequency of IPIs in patients receiving corticosteroid drugs worldwide. In this study, $19.6 \%$ of corticosteroid-treated individuals were positive for IPIs. This infection rate is lower than that previously reported in a study conducted in Egypt (92.3\%) among children chronically treated with corticosteroids [23].

Our findings showed that $S$. stercoralis, with a prevalence rate of $4.9 \%$, was a common intestinal parasite among the study population. Multiple epidemiological studies have illustrated that strongyloidiasis is common in the northern and southern regions of Iran $[6,9,10,24]$, which is related to the temperate climate and the high humidity of these regions. The infection rate in endemic areas of Iran varies according to the target population and diagnostic methods employed. Most studies conducted in Iran were community-based and in immunocompetent populations [6,10,24,25]. The prevalence of $S$. stercoralis in the current study was similar to that found in a previous study carried out on rural inhabitants of Mazandaran Province using the APC technique (4.9\%) [6]. Saeidinia et al. [24] reported a prevalence rate of $1.2 \%$ for S. stercoralis in institutionalized mentally disabled individuals in Guilan Province. Furthermore, a prevalence of $42 \%$ was reported for S. stercoralis among patients with eosinophilia in Guilan Province [26]. Another study among the residents of rehabilitation centers found 
an infection rate of $2.1 \%$ for S. stercoralis in Mazandaran Province [27]. Recently, the prevalence rate of this parasite was detected in 9.7\% of residents of Khouzestan Province using nested polymerase chain reaction [10]. Despite the high prevalence of strongyloidiasis in endemic regions of Iran, there still remains no comprehensive understanding of its epidemiology and seroepidemiology in immunocompromised patients. Recently, Rafiei et al. [13] detected anti-S. stercoralis antibodies in $14.4 \%$ of immunocompromised patients in southwest Iran.

Several epidemiological studies in different groups of immunocompromised patients across the world have reported S. stercoralis using parasitological methods. The prevalence rate of this parasite in our study was higher than those reported previously for northeast India (3.2\%) [28], Colombia (3.6\%) [29], Brazil (2.4\%) [30], and Saudi Arabia (2.2\%) [31] when studied among various groups of immunocompromised patients. S. stercoralis was reported in $4.4 \%$ of cancer patients undergoing chemotherapy in southern Brazil [21]. Blatt et al. [32] reported that $10 \%$ of HIV-positive individuals were infected with $S$. stercoralis in Brazil.

In the current study, the prevalence rate of $S$. stercoralis among users of corticosteroid drugs was higher than that among cancer patients; however, the difference between the 2 groups was not statistically significant. Glucocorticoids, such as hydrocortisone, prednisolone, methylprednisolone, betamethasone, and dexamethasone, suppress eosinophilia and lymphocyte activation and reduce inflammation, thereby impairing the ability of the intestine to contain parasites $[8,16]$. Furthermore, some researchers have suggested that these drugs may directly affect parasites, accelerating the transformation from rhabditiform to invasive filariform larvae $[8,16]$.

Our study showed that $S$. stercoralis infection was more prevalent in patients over 60 years of age than in other groups. This finding agrees with some previous studies that found a correlation between a higher prevalence rate of strongyloidiasis and increasing age $[9,10,33]$. This relationship may be attributed to the possibility of $S$. stercoralis autoinfections in infected individuals for several decades or even their entire lives. Furthermore, susceptibility to infection increases among older individuals due to the reduced efficiency of their immune response [34].

Our data showed that there was no significant relationship between S. stercoralis infection and sex. This finding is in agreement with some epidemiological studies $[10,13,35]$. However, several researchers reported that the rate of infection in males was higher than females due to a higher exposure of males to the source of infection as the result of working in rice and tea fields and gardening $[9,33]$. Other tested risk factors, including educational status, occupation, and location, were not associated with strongyloidiasis.

The application of a sensitive test for the diagnosis of strongyloidiasis is essential for high-risk individuals to decrease the mortality and morbidity associated with this infection. Several parasitological methods such as FEC, APC, Harada-Mori culture, and the Baermann method have been used to detect S. stercoralis larvae in stool samples [36]. Many studies have illustrated that APC is more sensitive than other parasitological methods for the diagnosis of S. stercoralis [6,7,36-38]. Our findings also confirmed that the sensitivity of APC was higher than that of other conventional methods. This culture protocol detected the parasite 3.4 times and 1.7 times more frequently than the direct wet-mount and FEC techniques.

In the present study, $B$. hominis was the most common infection in patients receiving immunosuppressive drugs (10.3\%). This is similar to the findings of other studies that detected B. hominis as the most common parasite among cancer patients in Iran (22.3\%) [19], immunosuppressed patients in Saudi Arabia (33.3\%) [39], and immunosuppressed patients in Iran (4.2\%) [17]. However, $B$. hominis is one of the most common parasites routinely found in human stool samples, and its pathogenicity is still highly controversial [40]. There are many potential sources for human infections, such as water resources, pets, and vegetables [41,42]. Its high prevalence is probably related to poor hygiene and the consumption of contaminated food and drinking water. In this study, the prevalence rate of this parasite in corticosteroid-treated patients was significantly higher than that in cancer patients.

Our results demonstrated a low-frequency rate of Giardia lamblia $(0.8 \%)$. This protozoan is more common in children than in adults [43], and our study participants were over 40 years old; therefore, such a finding was expected. Epidemiological studies among cancer patients in Iran revealed that the frequency of G. lamblia was between $0.4 \%$ and $5.1 \%[18,44,45]$.

In our study, 1 case of infection with Trichostrongylus spp. was observed. According to recent studies, human trichostrongyliasis is prevalent in northern Iran due to close contacts between people and herbivorous animals [46-50].

Our study showed that S. stercoralis is one of the most common parasites among patients receiving immunosuppressive drugs in northern Iran. Early diagnosis and proper treatment of patients before chemotherapy or steroid therapy are necessary to minimize the complications of severe strongyloidiasis. Therefore, utilizing sensitive diagnostic methods such as APC for patients at risk, with careful attention to the elderly, in endemic areas will prevent the potentially fatal consequences of this nematode.

\section{CONFLICT OF INTEREST}

The authors have no conflicts of interest to declare for this study.

\section{FUNDING}

This study has been fnancially supported by Research Deputy of Guilan University of Medical Sciences, with project No. 96050704.

\section{ACKNOWLEDGEMENTS}

The authors would like to thank all people who have contributed to this research. 


\section{AUTHOR CONTRIBUTIONS}

Conceptualization: MS, LM, KA. Data curation: MS, LM. Formal analysis: ZAR. Funding acquisition: MS. Methodology: MS, LM, ISM, BR, MRM, FS. Project administration: MS. Visualization: MS, LM, KA, ISM, BR, MRM, FS. Writing - original draft: MS, HM, FS. Writing - review \& editing: MS, HM, KA, FS, MRM, LM, ZAR, BR, ISM.

\section{ORCID}

Leila Mirzaei: https://orcid.org/0000-0003-4716-1162; Keyhan Ashrafi: https://orcid.org/0000-0002-1742-1245; Zahra Atrkar Roushan: https://orcid.org/0000-0001-8687-1242; Mohammad Reza Mahmoudi: https://orcid.org/0000-0001-6820-1288; Irandokht Shenavar Masooleh: https://orcid.org/0000-0002-3967-7022; Behnaz Rahmati: https://orcid.org/0000-0001-8556-9439; Farshid Saadat: https://orcid.org/0000-0002-5457-4195; Hamed Mirjalali: https:// orcid.org/0000-0002-2568-9022; Meysam Sharifdini: https://orcid. org/0000-0003-2686-7748

\section{REFERENCES}

1. Bundy DA, Hall A, Medley GF, Savioli L. Evaluating measures to control intestinal parasitic infections. World Health Stat Q 1992; 45:168-179.

2. Barsoum RS. Parasitic infections in transplant recipients. Nat Clin Pract Nephrol 2006;2:490-503.

3. Bisoffi Z, Buonfrate D, Montresor A, Requena-Méndez A, Muñoz J, Krolewiecki AJ, et al. Strongyloides stercoralis: a plea for action. PLoS Negl Trop Dis 2013; 7:e2214.

4. Grove DI. Human strongyloidiasis. Adv Parasitol 1996;38:251309.

5. Segarra-Newnham M. Manifestations, diagnosis, and treatment of Strongyloides stercoralis infection. Ann Pharmacother 2007; 41:1992-2001.

6. Kia EB, Mahmoudi M, Zahabioun F, Memar A. An evaluation on the efficacy of agar plate culture for detection of Strongyloides stercoralis. Iran J Parasitol 2007;2:29-34.

7. Sharifdini M, Mirhendi H, Ashrafi K, Hosseini M, Mohebali M, Khodadadi H, et al. Comparison of nested polymerase chain reaction and real-time polymerase chain reaction with parasitological methods for detection of Strongyloides stercoralis in human fecal samples. Am J Trop Med Hyg 2015;93:1285-1291.

8. Keiser PB, Nutman TB. Strongyloides stercoralis in the immunocompromised population. Clin Microbiol Rev 2004;17:208-217.

9. Sharifdini M, Kia EB, Ashrafi K, Hosseini M, Mirhendi H, Mohebali $\mathrm{M}$, et al. An analysis of clinical characteristics of Strongyloides stercoralis in 70 indigenous patients in Iran. Iran J Parasitol 2014;9:155-162.

10. Sharifdini M, Keyhani A, Eshraghian MR, Beigom Kia E. Molecular diagnosis of strongyloidiasis in a population of an endemic area through nested-PCR. Gastroenterol Hepatol Bed Bench
2018;11:68-74.

11. Esmaeli S, Fakhar M, Gohardehi S, Janbabaei Q, Ahmadpour E, Bastani R. Strongyloides stercoralis infection: neglected parasitic infection among cancer patients. J Jahrom Univ Med Sci 2012;10: 13-18.

12. Meamar AR, Rezaian M, Mohraz M, Hadighi R, Kia EB. Strongyloides stercoralis hyper-infection syndrome in HIV+/AIDS patients in Iran. Parasitol Res 2007;101:663-665.

13. Rafiei R, Rafiei A, Rahdar M, Keikhaie B. Seroepidemiology of Strongyloides stercoralis amongst immunocompromised patients in Southwest Iran. Parasite Epidemiol Control 2016;1:229-232.

14. Ebrahimi E, Bayat H, Neyshaburi MR, Zare Abyaneh H. Prediction capability of different soil water retention curve models using artificial neural networks. Arch Agron Soil Sci 2014;60:859-879.

15. Kazancı N, Gulbabazadeh T, Leroy SA, Ileri Ö. Sedimentary and environmental characteristics of the Gilan-Mazenderan plain, northern Iran: influence of long-and short-term Caspian water level fluctuations on geomorphology. J Mar Syst 2004;46:145-168.

16. Romano MC, Jiménez P, Miranda-Brito C, Valdez RA. Parasites and steroid hormones: corticosteroid and sex steroid synthesis, their role in the parasite physiology and development. Front Neurosci 2015;9:224.

17. Rasti S, Hassanzadeh M, Hooshyar H, Momen-Heravi M, Mousavi SG, Abdoli A. Intestinal parasitic infections in different groups of immunocompromised patients in Kashan and Qom cities, central Iran. Scand J Gastroenterol 2017;52:738-741.

18. Mohammadi-Ghalehbin B, Pezeshki A, Kohansal MH, Esmaeilnezhad G. Frequency of intestinal parasites in patients with malignancy in Ardabil province, northwest Iran. J Hum Environ Health Promot 2017;2:118-124.

19. Esteghamati A, Khanaliha K, Bokharaei-Salim F, Sayyahfar S, Ghaderipour M. Prevalence of intestinal parasitic infection in cancer, organ transplant and primary immunodeficiency patients in Tehran, Iran. Asian Pac J Cancer Prev 2019;20:495-501.

20. Al-Qobati SA, Al-Maktari MT, Al-Zoa AM, Derhim M. Intestinal parasitosis among Yemeni patients with cancer, Sana'a, Yemen. J Egypt Soc Parasitol 2012;42:727-734.

21. Jeske S, Bianchi TF, Moura MQ, Baccega B, Pinto NB, Berne ME, et al. Intestinal parasites in cancer patients in the South of Brazil. Braz J Biol 2018;78:574-578.

22. Abdel-Magied AA, El-Ghanam WA, El-Nemr HI, El-Henawy AA. Prevalence of intestinal parasites in cancer therapy recipients with concurrent diarrhea. Int J Trop Dis Health 2016;15:1-7.

23. Abdel-Hafeez EH, Ahmad AK, Ali BA, Moslam FA. Opportunistic parasites among immunosuppressed children in Minia District, Egypt. Korean J Parasitol 2012;50:57-62.

24. Saeidinia A, Tavakoli I, Naghipour MR, Rahmati B, Ghavami Lahiji $\mathrm{H}$, Salkhori O, et al. Prevalence of Strongyloides stercoralis and other intestinal parasites among institutionalized mentally disabled individuals in Rasht, northern Iran. Iran J Parasitol 2016; 11:527-533.

25. Shokri A, Sarasiabi KS, Teshnizi SH, Mahmoodi H. Prevalence of Strongyloides stercoralis and other intestinal parasitic infections 
among mentally retarded residents in central institution of southern Iran. Asian Pac J Trop Biomed 2012;2:88-91.

26. Ashrafi K, Tahbaz A, Rahmati B. Strongyloides stercoralis: the most prevalent parasitic cause of eosinophilia in Gilan Province, northern Iran. Iran J Parasitol 2010;5:40-47.

27. Ahmadi M, Beigom Kia E, Rezaeian M, Hosseini M, Kamranrashani B, Tarighi F. Prevalence of Strongyloides stercoralis and other intestinal parasites in rehabilitation centers in Mazandaran Province, northern Iran. J Mazandaran Univ Med Sci 2015;25: 1-7 (Persian).

28. Bora I, Dutta V, Lyngdoh WV, Khyriem AB, Durairaj E, Phukan AC. Study of intestinal parasites among the immunosuppressed patients attending a tertiary-care center in Northeast India. Int J Med Sci Pub Health 2016;5:924-929.

29. Botero JH, Castaño A, Montoya MN, Ocampo NE, Hurtado MI, Lopera MM. A preliminary study of the prevalence of intestinal parasites in immunocompromised patients with and without gastrointestinal manifestations. Rev Inst Med Trop Sao Paulo 2003;45:197-200.

30. de Paula FM, de Castro E, Gonçalves-Pires Md, Marçal Md, Campos DM, Costa-Cruz JM. Parasitological and immunological diagnoses of strongyloidiasis in immunocompromised and non-immunocompromised children at Uberlândia, State of Minas Gerais, Brazil. Rev Inst Med Trop Sao Paulo 2000;42:51-55.

31. Al-Megrin WA. Intestinal parasites infection among immunocompromised patients in Riyadh, Saudi Arabia. Pak J Biol Sci 2010;13: 390-394.

32. Marchi Blatt J, Cantos GA. Evaluation of techniques for the diagnosis of Strongyloides stercoralis in human immunodeficiency virus (HIV) positive and HIV negative individuals in the city of Itajaí, Brazil. Braz J Infect Dis 2003;7:402-408.

33. Khieu V, Schär F, Forrer A, Hattendorf J, Marti H, Duong S, et al. High prevalence and spatial distribution of Strongyloides stercoralis in rural Cambodia. PLoS Negl Trop Dis 2014;8:e2854.

34. Gardner ID. The effect of aging on susceptibility to infection. Rev Infect Dis 1980;2:801-810.

35. Luvira V, Trakulhun K, Mungthin M, Naaglor T, Chantawat N, Pakdee W, et al. Comparative diagnosis of strongyloidiasis in immunocompromised patients. Am J Trop Med Hyg 2016;95:401404.

36. Siddiqui AA, Berk SL. Diagnosis of Strongyloides stercoralis infection. Clin Infect Dis 2001;33:1040-1047.

37. Koga K, Kasuya S, Ohtomo H. How effective is the agar plate method for Strongyloides stercoralis? J Parasitol 1992;78:155-156.

38. de Kaminsky RG. Evaluation of three methods for laboratory di- agnosis of Strongyloides stercoralis infection. J Parasitol 1993;79: 277-280.

39. Faidah HS, Zaghlool DA, Soltane R, Elsayed FM. Opportunistic intestinal protozoal infections among immunosuppressed patients in a tertiary hospital Makkah, Saudi Arabia. J Health Sci 2016;6: 48-52.

40. Basak S, Rajurkar MN, Mallick SK. Detection of Blastocystis hominis: a controversial human pathogen. Parasitol Res 2014;113: 261-265.

41. Javanmard E, Rahimi HM, Niyyati M, Aghdaei HA, Sharifdini M, Mirjalali H, et al. Molecular analysis of Blastocystis sp. and its subtypes from treated wastewater routinely used for irrigation of vegetable farmlands in Iran. J Water Health 2019;17:837-844.

42. Mohammad Rahimi H, Nemati S, Mirjalali H, Sharifdini M, Zali MR. Molecular characterization and identification of Blastocystis and its subtypes from raccoon (Procyon lotor) in north of Iran. Parasitol Res 2020;119:2741-2745.

43. Thompson RA. Giardiasis: modern concepts in control and management. Ann Nestle Eng 2008;66:23-29.

44. Zali MR, Mehr AJ, Rezaian M, Meamar AR, Vaziri S, Mohraz M. Prevalence of intestinal parasitic pathogens among HIV-positive individuals in Iran. Jpn J Infect Dis 2004;57:268-270.

45. Zabolinejad N, Berenji F, Bayati Eshkaftaki E, Badeii Z, Banihashem A, Afzalaqaei M. Intestinal parasites in children with lymphohematopoietic malignancy in Iran, Mashhad. Jundishapur J Microbiol 2013;6:e7765.

46. Ashrafi K, Tahbaz A, Sharifdini M, Mas-Coma S. Familial Trichostrongylus infection misdiagnosed as acute fascioliasis. Emerg Infect Dis 2015;21:1869-1870.

47. Ghanbarzadeh L, Saraei M, Kia EB, Amini F, Sharifdini M. Clinical and haematological characteristics of human trichostrongyliasis. J Helminthol 2019;93:149-153.

48. Sharifdini M, Derakhshani S, Alizadeh SA, Ghanbarzadeh L, Mirjalali H, Mobedi I, et al. Molecular identification and phylogenetic analysis of human Trichostrongylus species from an endemic area of Iran. Acta Trop 2017;176:293-299.

49. Sharifdini M, Heidari Z, Hesari Z, Vatandoost S, Kia EB. Molecular phylogenetics of Trichostrongylus species (Nematoda: Trichostrongylidae) from humans of Mazandaran province, Iran. Korean J Parasitol 2017;55:279-285.

50. Ashrafi K, Sharifdini M, Heidari Z, Rahmati B, Kia EB. Zoonotic transmission of Teladorsagia circumcincta and Trichostrongylus species in Guilan province, northern Iran: molecular and morphological characterizations. BMC Infect Dis 2020;20:28. 